\title{
ANÁLISE COMPARATIVA HISTOPATOLÓGICA ENTRE A HÉRNIA DE DISCO CONTIDA E EXTRUSA
}

\author{
COMPARATIVE HISTOPATHOLOGIC ANALYSIS OF CONTAINED AND EXTRUDED \\ DISC HERNIATION
}

\author{
ANÁLISIS COMPARATIVO HISTOPATOLÓGICO ENTRE HERNIA DISCAL CONTENIDA \\ Y EXTRUIDA
}

Luciano Miller Reis Rodrigues ${ }^{1}$, Thérèse Theodoro Rachell ${ }^{2}$, Ana Maria Mader ${ }^{3}$, Carlo Milanil ${ }^{1}$ Fabrício Hidetoshi Ueno', Maria Aparecida da Silva Pinhal ${ }^{2}$

\begin{abstract}
RESUMO
Objetivo: Nosso estudo tem o objetivo de estudar as alterações histopatológicas, tais como neovascularização, infiltrado inflamatório, celularidade, apoptose, degeneração mucoide, alterações granulares e calcificação presentes nos tipos de hérnia (contida e extrusa), e também avaliar essas diferenças entre o núcleo pulposo e ânulo fibroso. Métodos: Foram analisados 65 discos lombares, os quais foram divididos em três grupos: hérnia extrusa com 25 casos, hérnia contida com 28 casos e 12 discos sem alteração degenerativa. Os fragmentos removidos foram separados em ânulo fibroso e núcleo pulposo. Foi realizada análise semiquantitativa por microscopia óptica das alterações histopatológicas. Resultado: Em relação aos parâmetros avaliados na análise comparativa entre ânulo fibroso e núcleo pulposo não houve variação estatística significativa entre os grupos, o que mostra que ambas as regiões são semelhantes. A hérnia extrusa apresentou maior proporção de infiltrado inflamatório e neovascularização. As alterações degenerativas não apresentaram uma variação significante conforme o tipo de hérnia. Conclusões: Na hérnia de disco há uma relação entre neovascularização, infiltrado inflamatório e o tipo de hérnia. Não há diferença histopatológica em relação à porção do disco intervertebral analisada.
\end{abstract}

Descritores: Disco intervertebral; Deslocamento do disco intervertebral; Histologia.

\begin{abstract}
Objective: to investigate histopathological changes such as neovascularization, inflammatory infiltrate, cellularity, apoptosis, mucoid degeneration, granular changes, and calcification in contained and extruded disc herniations, and to compare these differences in the nucleus pulposus and annulus fibrosus. Methods: 65 lumbar discs were evaluated. These were divided in three groups: 25 cases of extruded herniated discs, 28 cases of contained herniated discs, and 12 cases of discs without degenerative changes. Fragments were removed and separated into annulus fibrosus and nucleus pulposus. Semi-quantitative analysis of histopathologic changes was carried out, using a microscope. Results: in the comparative analysis between annulus fibrosus and nucleus pulposus, no statistical differences were obtained between these groups, showing that both regions are similar. The extruded disc herniation presented a higher proportion of inflammatory infiltrate and neovascularization. Degenerative changes did not present significant variation in relation to disc herniation type. Conclusion: There is a relation in disc herniation between neovascularitazion, inflammatory infiltrate and type of disc herniation. There is no histopathologic difference in relation of the portion of intervertebral disc analyzed.
\end{abstract}

Keywords: Intervertebral disk; Intervertebral disk displacement; Histology.

\section{RESUMEN}

Objetivo: Nuestro estudio tiene el objetivo de analizar las alteraciones histopatológicas tales como neovascularización, infiltrado inflamatorio, celularidad, apoptosis, degeneración mucoide, alteraciones granulares y calcificación, presentes según los tipos de hernia (contenida y extruida), así como evaluar esas diferencias entre el núcleo pulposo y el anillo fibroso. Métodos: Se analizaron 65 discos lumbares, que se dividieron en tres grupos: hernia extruida en 25 casos, hernia contenida en 28 casos y 12 discos sin alteración degenerativa. Los fragmentos extraídos se separaron en anillo fibroso y núcleo pulposo. Se llevó a cabo un análisis semicuantitativo por microscopia óptica de las alteraciones histopatológicas. Resultado: Respecto a los parámetros evaluados en el análisis comparativo entre anillo fibroso y núcleo pulposo, no se produjo variación estadística significativa entre los grupos, lo cual muestra que ambas regiones son semejantes. La hernia extruida presentó una mayor proporción de infiltrado inflamatorio y neovascularización. Las alteraciones degenerativas no presentaron una variación significativa según el tipo de hernia. Conclusiones: En la hernia discal hay una relación entre neovascularización, infiltrado inflamatorio y tipo de hernia. No hay diferencia histopatológica respecto a la parte del disco intervertebral analizada.

Descriptores: Disco intervertebral; Desplazamiento del disco intervertebral; Histología.

1. Disciplina de Ortopedia eTraumatologia - Faculdade de Medicina do ABC

2. Departamento de Bioquímica - Faculdade de Medicina do ABC

3. Departamento de Patologia - Faculdade de Medicina do ABC

Trabalho realizado na Faculdade de Medicina do ABC - SP - Brasil.

Endereço para Correspondência: Rua Tucuma, 199 - São Paulo - SP - Brasil - CEP: 01455-010 - E mail: luciano.miller@uol.com.br 


\section{INTRODUÇÃO}

A lesão do disco intervertebral é a causa mais comum de dor lombar e ciatalgia ${ }^{1}$. Na degeneração do disco intervertebral ocorrem lacerações no ânulo fibroso. Dependendo do grau de lesão do ânulo fibroso há uma perda da contenção do gel que forma a parte central do disco intervertebral, ocorrendo a formação da hérnia de disco ${ }^{2}$. Em relação à morfologia da hérnia de disco podemos observar fragmentos contidos ou subligamentares, onde não há a ruptura do ligamento longitudinal posterior, e fragmentos extrusos ou não contidos. No tecido da hérnia de disco as células inflamatórias migram através do endotélio dos vasos neoformados, sendo esse um dos fatores relacionados com a intensidade de dor nos pacientes com lesão discal ${ }^{3,4}$. O edema intraneural causado pela pressão e duração da compressão foi também relacionado com o processo álgico causado pela hérnia de disco ${ }^{5,6}$.

Diversas alterações histológicas ocorrem durante a degeneração do disco intervertebral. Boos et al. ${ }^{7}$ propuseram uma classificação semiquantitativa, correlacionando as alterações degenererativas apresentadas com a idade, com a classificação macroscópica de Thompson ${ }^{8}$. Nesse estudo não há uma análise do processo inflamatório e neovascularização.

A composição do fragmento herniário (núcleo pulposo, ânulo fibroso e placa terminal) varia de acordo com a idade, sendo essa alteração também relacionada com o quadro álgico do paciente com hérnia de disco ${ }^{9}$. A sintomatolgia relaciona-se com a localização, tamanho e composição do fragmento. Willburger et al. ${ }^{10}$ demonstraram que a sintomatologia clínica relacionava-se com a composição do fragmento discal, sendo que, no grupo onde a placa terminal era preponderante, a duração e o nível de dor eram mais intensos, colocando, como hipótese, a dificuldade de reabsorção do fragmento.

Apesar de diversas publicações analisarem a hérnia de disco, faltam estudos relacionando as alterações histológicas degenerativas com infiltrado inflamatório e presença de neovascularização. Também há pouca discussão relacionando o tipo de hérnia contida ou extrusa com esses fatores descritos acima. Nosso estudo tem como objetivo avaliar as alterações histológicas e relacionar o tipo de hérnia com o processo inflamatório e a neovascularização.

\section{MÉTODOS}

Analisamos 65 discos lombares retirados durante procedimento cirúrgico para descompressão da raiz nervosa. Todos os pacientes estudados tinham menos de 40 anos. O tempo de sintomatologia variou entre 4 semanas até 6 meses para indicação de procedimento cirúrgico.

Os discos analisados foram divididos em dois grupos: o de hérnia extrusa, com 28 casos, e o de hérnia contida, com 25 casos (Tabela 1). A classificação do tipo de hérnia foi feita com base em imagens obtidas pela ressonância magnética, observação visual durante o ato cirúrgico e análise da integridade do ligamento longitudinal posterior.

Tabela 1. Análise descritiva geral.

\begin{tabular}{c|c|c}
\hline Variável & N & $\%$ \\
\hline Pacientes & 65 & 100 \\
\hline Patologia & $\mathbf{N}$ & $\%$ \\
\hline Hérnia Contida & 28 & 43 \\
\hline Hérnia Extrusa & 25 & 38,5 \\
\hline Fratura & 12 & 18,5 \\
\hline Região & $\mathbf{N}$ & $\%$ \\
\hline Núcleo Pulposo & 50 & 77 \\
\hline Ânulo Fibroso & 25 & 23 \\
\hline
\end{tabular}

Os discos abordados foram L4-L5 e L5-S1. O procedimento teve como objetivo a liberação da raiz nervosa realizada através da microdiscectomia. Foram analisados 12 discos normais, pela classificação de Pfirrmann, obtidos de pacientes que sofreram fratura da coluna lombar ${ }^{11}$.

Os fragmentos removidos foram separados em ânulo fibroso e núcleo pulposo. Posteriormente cada fragmento foi classificado quantitativamente quanto às alterações degenerativas pela classificação de Boss et al. ${ }^{7}$ nos itens: celularidade, morte celular, degeneração mucóide, calcificação. Além desses itens, incluímos na nossa análise a verificação de infiltrado inflamatório e a presença de neovascularização.

Foi realizada a avaliação de dor através da escala analógica visual, e somente os pacientes que apresentavam pontuação maior que oito foram incluídos no nosso protocolo. No exame físico todos apresentaram lasègue positivo. Em relação à alteração neurológica, 31 referiam mudança de sensibilidade e, 18, diminuição de força muscular no dermátomo correspondente.

\section{RESULTADO}

Não houve variação estatística significante comparando o núcleo pulposo e o ânulo fibroso quanto às alterações degenerativas, presença de vasos e infiltrado inflamatório (Tabela 2).

No grupo de pacientes com hérnia extrusa, em 84\% foi obser-

Tabela 2. Análise descritiva por grupo de patologia.

\begin{tabular}{|c|c|c|c|c|}
\hline & $\begin{array}{l}\text { Hérnia Contida } \\
\text { (N/\%) }\end{array}$ & $\begin{array}{c}\text { Hérnia } \\
\text { Extrusa (N/\%) }\end{array}$ & $\begin{array}{c}\text { Fratura } \\
(\mathrm{N} / \%)\end{array}$ & $\mathbf{P}$ \\
\hline $\begin{array}{c}\text { Região } \\
\text { Núcleo Pulposo } \\
\text { Ânulo Fibroso }\end{array}$ & $\begin{array}{c}22 / 78,6 \\
6 / 21,4\end{array}$ & $\begin{array}{c}20 / 80,0 \\
5 / 20,0\end{array}$ & $\begin{array}{l}8 / 66,7 \\
4 / 33,3\end{array}$ & 0.642 \\
\hline $\begin{array}{c}\text { Vasos } \\
\text { Negativo } \\
\text { Positivo + } \\
\text { Positivo ++ }\end{array}$ & $\begin{array}{c}27 / 96,4 \\
1 / 3,6 \\
0 / 0\end{array}$ & $\begin{array}{c}4 / 16,0 \\
15 / 60,0 \\
6 / 24,0\end{array}$ & $\begin{array}{c}12 / 100 \\
0 / 0 \\
0 / 0\end{array}$ & $<0.001$ \\
\hline $\begin{array}{c}\text { Infiltrado } \\
\text { Negativo } \\
\text { Positivo + } \\
\text { Positivo ++ }\end{array}$ & $\begin{array}{c}27 / 96,4 \\
1 / 3,6 \\
0 / 0\end{array}$ & $\begin{array}{c}8 / 32,0 \\
14 / 56,0 \\
3 / 12,0\end{array}$ & $\begin{array}{c}12 / 100 \\
0 / 0 \\
0 / 0\end{array}$ & $<0.001$ \\
\hline $\begin{array}{c}\text { Celularidade } \\
\text { Positivo + } \\
\text { Positivo ++ } \\
\text { Positivo +++ }\end{array}$ & $\begin{array}{c}9(32,1) \\
13(46,4) \\
6(21,4)\end{array}$ & $\begin{array}{c}6 / 24,0 \\
14 / 56,0 \\
5 / 20,0\end{array}$ & $\begin{array}{c}0 / 0 \\
12 / 100 \\
0 / 0\end{array}$ & 0.034 \\
\hline $\begin{array}{c}\text { Apoptose } \\
\text { Positivo + } \\
\text { Positivo ++ } \\
\text { Positivo +++ }\end{array}$ & $\begin{array}{c}4 / 14,3 \\
14 / 50,0 \\
10 / 35,7\end{array}$ & $\begin{array}{c}9 / 36,0 \\
10 / 40,0 \\
6 / 24,0 \\
\end{array}$ & $\begin{array}{c}0 / 0 \\
5 / 41,7 \\
7 / 58,3 \\
\end{array}$ & 0.059 \\
\hline $\begin{array}{c}\text { Degeneração } \\
\text { Mucóide } \\
\text { Positivo + } \\
\text { Positivo ++ } \\
\text { Positivo +++ }\end{array}$ & $\begin{array}{c}1 / 3,6 \\
18 / 64,3 \\
9 / 32,1\end{array}$ & $\begin{array}{c}0 / 0 \\
13 / 52,0 \\
12 / 48,0\end{array}$ & $\begin{array}{c}6 / 50,0 \\
5 / 41,7 \\
1 / 8,3\end{array}$ & $<0.001$ \\
\hline $\begin{array}{c}\text { Calcificação } \\
\text { Negativo } \\
\text { Positivo + } \\
\text { Positivo ++ }\end{array}$ & $\begin{array}{c}13 / 46,4 \\
7 / 25,0 \\
8 / 28,6\end{array}$ & $\begin{array}{l}11 / 44,0 \\
8 / 32,0 \\
6 / 24,0\end{array}$ & $\begin{array}{c}11 / 91,7 \\
1 / 8,3 \\
0 / 0\end{array}$ & 0.059 \\
\hline $\begin{array}{c}\text { Alterações } \\
\text { Granulares } \\
\text { Negativo } \\
\text { Positivo + } \\
\text { Positivo ++ } \\
\text { Positivo +++ }\end{array}$ & $\begin{array}{c}6 / 21,4 \\
7 / 25,0 \\
10 / 35,7 \\
5 / 17,9\end{array}$ & $\begin{array}{l}6 / 24,0 \\
8 / 32,0 \\
8 / 32,0 \\
3 / 12,0\end{array}$ & $\begin{array}{c}3 / 25,0 \\
9 / 75,0 \\
0 / 0 \\
0 / 0\end{array}$ & 0.055 \\
\hline
\end{tabular}


vada a presença de vasos com uma ou duas cruzes. No grupo de hérnia contida e fratura houve uma baixa presença de neovascularização. Também foi semelhante o resultado quanto à detecção de infiltrado inflamatório, onde os pacientes com hérnia contida (96,5\%) não apresentaram infiltrado inflamatório.

O grupo de fratura apresentou $100 \%$ dos pacientes com celularidade duas cruzes, enquanto o grupo de hérnia contida apresentou: $32 \%$ de celularidade uma cruz positiva, $46 \%$ de celularidade duas cruzes positivas e $21 \%$ de celularidade três cruzes positivas. No grupo de hérnia extrusa, foram observados $24 \%$ dos pacientes com celularidade uma cruz positiva, $56 \%$ com duas cruzes positivas e $20 \%$ com três cruzes positivas. A celularidade mostrou-se maior no grupo controle em comparação com o grupo de hérnia.

Quanto à degeneração mucóide, $50 \%$ dos casos de fratura apresentaram degeneração uma cruz, enquanto 100\% dos casos de hérnia extrusa apresentaram degeneração duas e três cruzes, sendo $50 \%$ dos casos de cada. Na hérnia contida, 64\% apresentaram degeneração duas cruzes.

$\mathrm{Na}$ calcificação não houve variação estatística significante. Com relação à presença de morte celular houve uma tendência de redução no grupo de hérnia extrusa comparando com os dois outros grupos. $\mathrm{Na}$ análise das alterações granulares, verificou-se na hérnia contida e extrusa uma maior tendência à apresentação de alterações, comparando com o grupo controle.

\section{DISCUSSÃO}

A composição do fragmento herniário tem sido estudada por diversos autores $^{12-14}$. O primeiro relato foi feito em 1934 por Mixter e Barr que descreveram a presença de anulo fibroso em quatro de 11 fragmentos herniários. Os diversos trabalhos realizados demonstraram que a composição do fragmento herniário é uma combinação de anulo fibroso, núcleo pulposo e placa terminal, diferenciando a composição conforme a idade e o mecanismo de trauma ${ }^{15}$. Nosso estudo demonstrou uma maior proporção de núcleo pulposo corroborando com o trabalho de Willburger et al. ${ }^{10}$.

Virri et al. ${ }^{16}$ observaram a grande presença de neovascularização no seguimento herniário, sugerindo ser uma tentativa de aumento da nutrição destes discos após a lesão tecidual. No seu artigo apenas relacionaram o tipo de hérnia extrusa ou contida. No presente estudo, além de estudarmos a presença de neovasculari- zação, demonstramos não haver diferença estatística em relação ao componente do disco (núcleo pulposo ou anulo fibroso) herniado. Com isso levantamos a hipótese que a matrix extracelular, após o desequilíbrio provocado pela lesão tecidual, influencia nessa vascularização, independente do componente discal.

A presença de infiltrado inflamatório ocorreu mais frequentemente em fragmentos extrusos, comparando com a hérnia contida. Yasuma et al. ${ }^{17}$ demonstraram que a ruptura do ligamento influência a migração de fatores inflamatórios e o aumento da vascularização, sendo esse último mais frequente na periferia do fragmento e anulo fibroso. Em nossa série de casos verificamos que não apenas o anulo fibroso apresenta aumento da vascularização e infiltrado inflamatório, mas também o núcleo pulposo. Isso mostra que a neovascularização está ocorrendo com o processo degenerativo.

A hérnia de disco pode variar consideravelmente em termos de características histológicas e mudanças estruturais da matrix extracelular ${ }^{18}$. A quebra da placa terminal na junção entre o disco é considerada a etapa inicial de lesão discal e início do processo de desenvolvimento da hérnia de disco ${ }^{19}$. A neovascularização inicia-se com a quebra da matrix extracelular em uma tentativa de cicatrização dessa lesão. Com essa desorganização a pressão no disco intervertebral pode propiciar uma ruptura desse disco e a formação do fragmento discal.

Houve uma tendência de diminuição da morte celular nos fragmentos extrusos comparando com o grupo controle. O destacamento do disco faz com que ocorra uma diminuição da constante reparação celular pela falta de contato com as outras regiões do disco. Isso corrobora com os achados de Yasuma et al. ${ }^{20}$ que demonstraram a influência do tempo na neovascularização em fragmentos extrusos, onde em mais de 6 meses ocorre uma baixa vascularização.

\section{CONCLUSÃO}

Na hérnia de disco há uma relação entre neovascularização e infiltrado inflamatório e o tipo de hérnia, mas esta é independente da porção do disco intervertebral. As alterações degenerativas do disco são um fator predisponente para a formação da hérnia de disco, mas isto é variável e dependente de fatores individuais e externos para definição do tipo de hérnia.

\section{REFERÊNCIAS}

1. Pearce J, Moll JM. Conservative treatment and natural history of acute lumbar disc lesions. J Neurol Neurosurg Pschiatry. 1967;30(1):13-7.

2. Hoogendoorn RJ, Wuisman PI, Smith TH, Everts VE, Helder MN. Experimental intervertebral disc degeneration induced by chondroitinase ABC in the goat. Spine (Phila Pa 1976). 2007;32(17):1816-25.

3. Franson RC, Saal JS, Saal JA. Human disc phospholipase A2 is inflammatory. Spine (Phila Pa 1976).1992; 17(6 Suppl):S129-32

4. McCarron RF, Wimpee MW, Hudkins PG, Laros GS. The inflammatory effect of nucleus pulposus: a possible element in the pathogenesis of low back pain. Spine (Phila Pa 1976). 1987;12(8):760-4.

5. Lind B, Massie JB, Lincoln T, Myers RR, Swenson MR, Garfin SR. The effects of induced hypertension and acute graded compression on impulse propagation in the spine nerve roots of the pig. Spine (Phila Pa 1976). 1993;18(121):1550-6.

6. Pedowitz RA, Garfin SR, Massie JB, Hargens AR, Swenson MR, Myers RR et al. Effects of magnitude and duration of compression on spinal nerve root conduction. Spine (Phila Pa 1976). 1992;17(2):194-9

7. Boos N, Weissbach S, Rohrbach H, Weiler C, Spratt KF, Nerlich AG. Classfication of age-related changes in lumbar discs:2002 Volvo award in basic science. Spine (Phila Pa 1976).2002;27(23):2631-44

8. Thompson JP, Pearce RH, Schechter MT, Adams ME, Tsang IK, Bishop PB. Preliminary evaluation of a scheme for grading the gross morphology of the human intervertebral disc. Spine (Phila Pa 1976). 1990;15(5):411-5.

9. Eckert C, Decker A. Pathological studies of intervertebral discs. J Bone Joint Surg Am. 1947;29(2):447-54.

10. Willburger RE, Ehiosun UK, Kuhnen C, Krämer J, Schmid G. Clinical symptoms in lumbar

disc herniations and their correlation to the histological composition of extruded disc material. Spine (Phila Pa 1976). 2004;29(15):1655-61.

11. Pfirrmann CW, Metzdorf A, Zanetti M, Hodler J, Boos N. Magnetic resonanceclassification of lumbar intervertebral disc degeneration. Spine (Phila Pa 1976). 2001;26(17):1873-8.

12. Brock M, Patt S, Mayer HM. The form and structure of the extruded disc.Spine (Phila Pa 1976). 1992;17(12):1457-61.

13. Harada $Y$, Nakahara S. A pathologic study of lumbar disc herniation in the eldery. Spine (Phila Pa 1976). 1989;14(9):1020-4.

14. Lipson S. Metaplastic proliferative fibrocartilage as an alternative concept to herniated intervertebral disc. Spine (Phila Pa 1976). 1988;13(9):1055-60.

15. Veres SP, Robertson PA, Broom ND. The morphology of acute disc herniation: a clinically relevant model defining the role of flexion. Spine (Phila Pa 1976). 2009;34(21):2288-96.

16. Virri J, Grönblad M, Savikko J, Palmgren T, Seitsalo S, Ruuskanen M et al. Prevalence, morphology, and topography of blood vessels in herniated disc tissue: a comparative immunocytochemical study. Spine (Phila Pa 1976). 1996;21(16):1856-63.

17. Yasuma T, Arai K, Yamauchi Y. The histology of lumbar intervertebral disc herniation The significance of small blood vessels in the extruded tissue. Spine (Phila Pa 1976).1993;18(13):1761-5.

18. Roberts S, Evans H, Trivedi J, Menage J: Histology and pathology of humanintervertebral disc. J Bone Joint Surg Am. 2006;88 (2 Suppl):10-4.

19. Kobuko Y, Kobayashi S, Uchida K, Noriki S, Imamura Y, Furusawa N et al. Herniated and spondylotic intervertebral discs of the human cervical spine: histological and immunohistological observations. Acta Histochem Cytochem.2004;37(2):109-4.

20. Yasuma T, Makino E, Saito S, Inui M. Histological development of intervertebral disc herniation. J Bone Joint Surg Am. 1986;68(7):1066-77. 